\title{
Assessing the reliability of DP and Fermi estimates in single and dual bolus cardiac MR perfusion imaging
}

\author{
Giorgos Papanastasiou ${ }^{1,2^{*}}$, Michelle C Williams², Shirjel Alam², Mark Dweck², Saeed Mirsadraee ${ }^{1}$, Calum D Gray ${ }^{1}$, \\ Martin Connell', Thomas J MacGillivray ${ }^{1}$, David Newby ${ }^{1,2}$, Scott Semple ${ }^{1,2}$
}

From 17th Annual SCMR Scientific Sessions

New Orleans, LA, USA. 16-19 January 2014

\section{Background}

MR dual bolus (DB) perfusion imaging has been effectively shown to eliminate signal saturation of the arterial input function (AIF) and allow more reliable quantification of myocardial (Myo) blood flow (MBF). Single bolus (SB) protocols are prone to AIF signal saturation but have been widely used in clinical studies. The Fermi model has been well established for MBF quantification. The distributed parameter (DP) model has been implemented in a recent study to measure additional physiological parameters (PP) such as intravascular (vb) and extravascular-extracellular space (ve) of the coronary arteries (CAs). Our study aims to a) validate absolute values, correlations and differences between $\mathrm{SB}$ versus $\mathrm{DB}$ estimates and $\mathrm{b}$ ) assess which model more reliably fits SB data.

\section{Methods}

After informed consent, 8 healthy volunteers underwent adenosine stress-rest $M R$ myocardial perfusion imaging (3T Verio, Siemens AG, Healthcare Sector). A dilute $(0.006 \mathrm{mmol} / \mathrm{kg}) \mathrm{Gd}$-based contrast agent (Gadovist, Bayer Healthcare) was injected to allow extraction of the AIF for the DB analysis followed by a standard (0.03 $\mathrm{mmol} / \mathrm{kg}$ ) Gd dose for the extraction of Myo signal intensity curves (Ishida $\mathrm{M}$ et al. 2011). Images were acquired in mid-diastole. SB AIF data were generated by extracting the AIF from the standard Gd dose component of the DB data. Signal intensity AIF and Myo curves were converted to Gd concentration curves (Biglands J et al. 2011). The Fermi model (Jerosch-Herold M et al. 1998) was used to quantify MBF as a reference standard against

${ }^{1}$ Clinical Research Imaging Center, University of Edinburgh, Edinburgh, Lothian, UK

Full list of author information is available at the end of the article
DP-MBF (Broadbent et al. 2013). DP and Fermi values were examined both in SB and DB data. Bland Altman plots and paired $t$-test ( $\mathrm{p}$ values $<0.01$ were considered significant) were used for data analysis.

\section{Results}

The most significant difference between SB and DB parameter estimates was observed in the Fermi model during stress (Table $1 \mathrm{p}<0.01$ ). Fermi values were influenced by saturation at the AIF peak because the Fermi model fits only to the first pass Myo curves (Figure 1a). There was no significant difference between DP-SB and DP-DB values: $\operatorname{MBF}(p=0.38, p=0.89)$, ve $(p=0.02, p=0.04)$ and permeability surface area product (PS) $(\mathrm{p}=0.11$, $\mathrm{p}=0.02)$, at stress and rest respectively. Our estimates of

Table 1 Parameter comparisons are shown in SB and DB data both at stress (S) and rest (R).

\begin{tabular}{ccccc}
\hline $\begin{array}{c}\text { Modeling values/ } \\
\text { Method }\end{array}$ & DP-DB & DP-SB & Fermi-DB & Fermi-SB \\
\hline MBF-S $(\mathrm{ml} / \mathrm{min} / \mathrm{ml})$ & $3.20(0.97)$ & $3.40(0.74)$ & $3.60(0.79)$ & $4.50(0.80)$ \\
\hline MBF-R (ml/min/ml) & $1.23(0.37)$ & $1.45(0.40)$ & $1.47(0.50)$ & $1.56(0.45)$ \\
\hline PS-S $(\mathrm{ml} / \mathrm{min} / \mathrm{ml})$ & $0.98(0.32)$ & $1.09(0.21)$ & & \\
\hline PS-R $(\mathrm{ml} / \mathrm{min} / \mathrm{ml})$ & $0.56(0.15)$ & $0.62(0.14)$ & & \\
\hline E-S & $0.45(0.04)$ & $0.45(0.03)$ & & \\
\hline $\mathrm{E}-\mathrm{R}$ & $0.56(0.04)$ & $0.54(0.03)$ & & \\
\hline $\mathrm{vb}-\mathrm{S}$ & $0.08(0.02)$ & $0.09(0.02)$ & & \\
\hline $\mathrm{vb}-\mathrm{R}$ & $0.04(0.01)$ & $0.04(0.01)$ & & \\
\hline $\mathrm{ve}-\mathrm{S}$ & $0.17(0.05)$ & $0.20(0.04)$ & & \\
\hline $\mathrm{ve}-\mathrm{R}$ & $0.17(0.06)$ & $0.20(0.05)$ & & \\
\hline
\end{tabular}

The extraction fraction $(\mathrm{E})$ and $\mathrm{vb}$ did not change in $\mathrm{SB}$ versus $\mathrm{DB}$ analysis: $\mathrm{E}-\mathrm{S}$ $(p=0.90), E-R(p=0.10)$, vb-S $(p=0.09)$, vb-R $(p=0.68)$. Mean values $( \pm S D)$ are shown. 


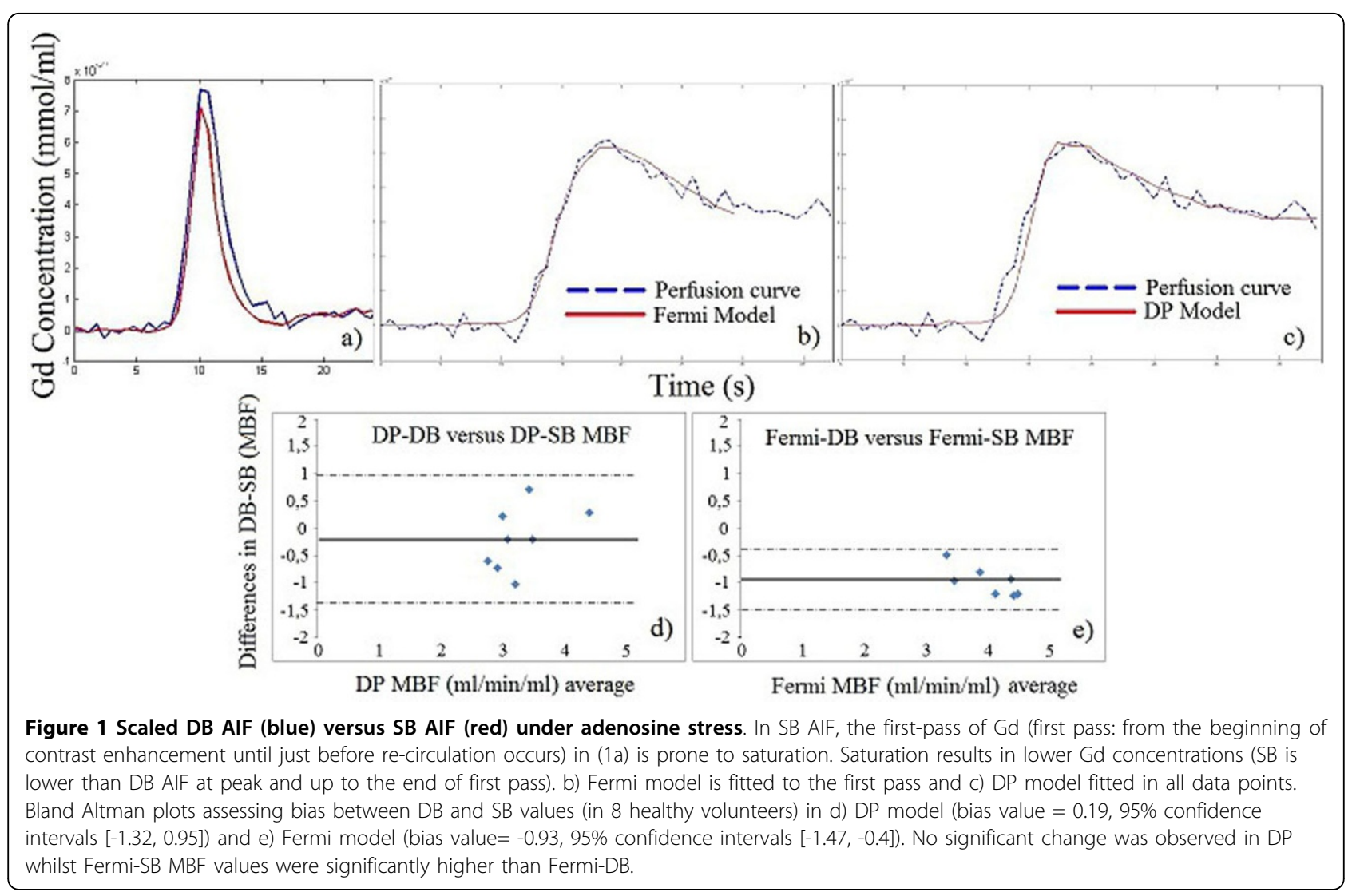

PS are lower than those reported by Broadbent et al's study which used mid-systolic data. This suggests that DP may be capable of detecting lower Gd permeability from vb into ve which is associated with relaxation of the subendocardial CAs during mid-diastole. In our study, DP was fitted for the first time in mid-diastole, which is the preferable method for perfusion acquisition.

\section{Conclusions}

DP-MBF and PP values were not significantly affected when SB AIF was used which suggests that DP model might more reliably model SB data than Fermi. This should be further investigated in larger populations. PS was low compared to previous mid-systole analysis, suggesting that DP may show potential in detecting decreased permeability of the CAs in mid-diastole.

\section{Funding}

This work was made possible through funding and continued support from the British Heart Foundation.

\section{Authors' details}

${ }^{1}$ Clinical Research Imaging Center, University of Edinburgh, Edinburgh, Lothian, UK. ${ }^{2}$ Centre for Cardiovascular Science, University of Edinburgh, Edinburgh, Lothian, UK.
Published: 16 January 2014

\section{doi:10.1186/1532-429X-16-S1-P347}

Cite this article as: Papanastasiou et al: Assessing the reliability of DP and Fermi estimates in single and dual bolus cardiac MR perfusion imaging. Journal of Cardiovascular Magnetic Resonance 2014 16(Suppl 1): P347.

\section{Submit your next manuscript to BioMed Central} and take full advantage of:

- Convenient online submission

- Thorough peer review

- No space constraints or color figure charges

- Immediate publication on acceptance

- Inclusion in PubMed, CAS, Scopus and Google Scholar

- Research which is freely available for redistribution

Submit your manuscript at www.biomedcentral.com/submit 